\title{
Arterial stiffness as a risk factor for hypotension during induction of general anaesthesia
}

Ianis Siriopol ${ }^{1,2}$, Ioana Grigoras ${ }^{1,2}$, Dimitrie Siriopol ${ }^{3,4}$, Adi Ciumanghel ${ }^{5}$, Daniel Rusu ${ }^{1}$, Mihaela Blaj ${ }^{1,5}$, Adrian Covic ${ }^{1,6}$

\author{
1"Grigore T. Popa” University of Medicine and Pharmacy, lasi, Romania \\ 2Department of Anaesthesia and Intensive Care, Regional Institute of Oncology, \\ Iasi, Romania \\ "3tefan cel Mare" University, Suceava, Romania \\ ${ }^{4}$ Department of Nephrology, "Sf. Ioan cel Nou" County Hospital, Suceava, Romania \\ ${ }^{5}$ Department of Anaesthesia and Intensive Care, "Sf. Spiridon" University Hospital, \\ Iasi, Romania \\ ${ }^{6}$ Department of Nephrology, “Dr. C. I. Parhon” University Hospital, Iasi, Romania
}

Submitted: 9 November 2020

Accepted: 26 January 2021

Arch Med Sci

DOI: https://doi.org/10.5114/aoms/132836

Copyright $\odot 2021$ Termedia \& Banach

\section{Abstract}

Introduction: Hypotension after induction of general anaesthesia is identified as an independent factor in predicting adverse clinical outcomes. Preoperative evaluation of arterial stiffness could identify patients with an impaired vascular function and an altered haemodynamic response to induction of general anaesthesia. The purpose of this study is to investigate the relationship between arterial stiffness and blood pressure variation during induction of general anaesthesia.

Material and methods: This was an observational study that included patients who underwent surgical procedures under general anaesthesia. We used several systolic arterial pressure and mean arterial pressure thresholds for defining hypotension. Both absolute thresholds and thresholds relative to a baseline blood pressure were chosen based on the most frequently used definitions. Patient carotid-femoral pulse wave velocity determination, preoperative preparation, and induction of general anaesthesia were standardized. Results: Our study included 115 patients. Both univariate and multivariate analysis showed that carotid-femoral pulse wave velocity was significantly associated with post-induction hypotension when defined as a systolic arterial pressure decrease of $>30 \%$ or $>40 \%$ from baseline or as a mean arterial pressure decrease of $>40 \%$ from baseline. Also, carotid-femoral pulse wave velocity was positively associated with duration of post-induction hypotension.

Conclusions: Preoperative assessment of arterial stiffness identifies patients at risk of a pronounced decrease in blood pressure during induction of general anaesthesia.

Key words: arterial stiffness, arterial blood pressure, hypotension, induction of anaesthesia, risk factor.

\section{Introduction}

Haemodynamic instability in patients undergoing surgery under general anaesthesia is highly prevalent and is associated with unfavourable patient outcome [1]. Several clinical studies demonstrated an association

\author{
Corresponding author: \\ Dimitrie Siriopol PhD \\ "Stefan cel Mare" University \\ University Street No 13 \\ 720229 \\ Suceava, Romania \\ E-mail: \\ dimitrie.siriopol@yahoo.com
}


between intraoperative hypotension $(\mathrm{IOH})$ and deleterious effects on organ function and integrity (myocardial injury, stroke, acute kidney injury, mesenteric hypoperfusion) in surgical patients [2, 3]. Maheshwari et al. reported that one-third of hypotensive events during elective non-cardiac surgery occurred before surgical incision [4]. Hypotension after induction of general anaesthesia is identified as an independent factor in predicting adverse clinical outcomes $[5,6]$.

Hypotension between anaesthetic induction and surgical incision is a largely preventable consequence of anaesthesia. Prevention of hypotensive events plays a key role in providing patient safety, taking into account that maintaining haemodynamic stability is essential for reduction of postoperative complications [7].

Prediction of $\mathrm{IOH}$ based on preoperatively identified risk factors remains a difficult task. Moreover, $\mathrm{IOH}$ lacks a clear definition. A 2007 systematic review identified 140 different definitions from 130 studies, with reported incidence of $\mathrm{IOH}$ between 5\% and $99 \%$ depending on which definition was used [1].

Arterial stiffness is an index of vascular health. It has been associated with cardiovascular diseases and mortality $[8,9]$ and represents a distinct feature of vascular disease [10]. Impairment of vascular function, such as increased arterial stiffness, is closely associated with the development and maintenance of atherosclerotic conditions, leading to target organ damage and cardiovascular complications [11]. Pulse wave velocity (PWV) is the most useful and robust index of arterial stiffness and is an important biomarker of vascular damage [12]. Pulse wave velocity is a functional parameter directly affected by arterial wall stiffness and is regarded as the in vivo "gold standard" index of arterial stiffness [13]. Carotid-to-femoral PWV (Cf-PWV) has been used as the most reliable surrogate for arterial stiffness [14].

Preoperative evaluation of arterial stiffness could identify patients with an impaired vascular function and at high risk of arterial hypotension at induction of general anaesthesia, resulting in an individualized approach. Post-induction hypotension (PIH) is a preventable event as arterial pressure is manageable by intravenous fluid, vasopressors, or adapting the anaesthetic technique. Identifying patients at risk, and prevention and early correction of $\mathrm{PIH}$ are essential parts of optimal anaesthesiology care.

The relationship between arterial stiffness and cardiovascular response to induction of general anaesthesia was evaluated in only a few trials, with conflicting results $[15,16]$.

The main goal of our study was to investigate the relationship between arterial stiffness and blood pressure changes during induction of general anaesthesia.

\section{Material and methods}

\section{Patients}

The study was performed in the Department of Anaesthesiology and Intensive Care of the Regional Institute of Oncology lasi, Romania between January and December 2017. The study protocol was reviewed and approved by the Ethics Committee of the University of Medicine and Pharmacy "Grigore T. Popa", Iasi (approval number 140724/2016). Prior to study enrolment, all patients signed an informed consent form, which included written information about the nature of the study, its aim, the expected advantages and possible risks, and also data protection assurances. The prospective observational study included patients scheduled for gastro-intestinal and gynaecological surgery under general anaesthesia. Inclusion criteria were as follows: 1) age more than 18 years, 2) elective non-cardiac surgery with an estimated duration of more than 1 hour, 3) general anaesthesia, and 4) tracheal intubation and mechanical ventilation during general anaesthesia. The following exclusion criteria were applied: 1) cardiac arrhythmia, 2) severe cardiac impairment (left ventricular ejection fraction < 30\%), 3) congenital heart disease, 4) American Society of Anesthesiologists (ASA) physical status IV or higher, 5) three or more attempts of endotracheal intubation, 6) insulin-treated diabetes mellitus, 7) chronic kidney disease on haemodialysis, 8) sepsis, and 9) uncorrected preoperative dehydration. The following baseline data were recorded for all patients: weight, height, body mass index, presence of comorbidities (blood hypertension, coronary artery disease (CAD), history of stroke, peripheral vascular disease, chronic kidney disease, diabetes mellitus, chronic liver disease), and use of chronic medication ( $\beta$-blockers, calcium-channel blockers, angiotensin-converting enzyme inhibitors [ACEI], angiotensin receptor blockers [ARB], diuretics, central antihypertensive drugs, oral antidiabetics). Angiotensin-converting enzyme inhibitors, $A R B$, and diuretics were discontinued on the day before surgery.

\section{Arterial stiffness evaluation}

The Cf-PWV was assessed on the day before surgery using the commercially available system SphygmoCorTM (PWV Inc., Westmead, Sydney, Australia).

The Cf-PWV were determined by a single trained operator. After explaining the procedure, body height and weight were measured. The patient, in supine position, connected to the ECG 
leads of the SphygmoCorTM, had 5 minutes of rest before blood pressure measurement was performed using a semi-automated oscillometric device. Using a tape measure, we manually assessed the distance from the suprasternal notch to the femoral artery (one detection point) and to the carotid artery (the other detection point) and introduced the data into the SphygmoCorTM system. The pressure wave forms were acquired at the carotid and femoral locations sequentially, using a pressure probe that serves to non-invasively detect the pressure waveform by applanation tonometry, and were synchronized on the ECG waveform. Carotid-to-femoral pulse wave velocity is calculated from measurements of pulse transit time and the distance travelled by the pulse between the sites of recording, data analysis being performed by a specially designed software.

We determined 2 Cf-PWV values for every patient and used the mean for further analysis. If the difference between these measurements was higher than $0.5 \mathrm{~m} / \mathrm{s}$, then another measurement was performed and the median was used for analysis.

\section{Induction of anaesthesia protocol}

Patient preoperative preparation and induction of general anaesthesia were standardized. No patient received premedication. The patients were permitted to drink clear liquids up to 2 hours before the surgery and a light meal up to 6 hours before the surgery. The intraoperative monitoring (Drager Primus ${ }^{\circledast}$ ) included the following: electrocardiography, pulse oximetry, non-invasive arterial blood pressure, bispectral index (BIS) for evaluation of depth of anaesthesia, end-tidal carbon dioxide, oxygen concentration, anaesthetic gas concentration, and neuromuscular block monitoring. The choice of anaesthetics drugs was standardized. General anaesthesia was induced with propofol $1.5-2.5 \mathrm{mg} / \mathrm{kg}$, fentanyl 2-3 $\mathrm{\mu g} /$ $\mathrm{kg}$, and rocuronium $0.4-0.6 \mathrm{mg} / \mathrm{kg}$. These drugs were administered at the same speed and within 60-90 seconds. Propofol was administered until loss of consciousness (as judged by loss of verbal contact with the patient) had been achieved. All patients were ventilated with oxygen and sevoflurane by a bag valve-mask for 3-5 minutes, maintaining a BIS score between 40 and 50. Airway was secured with tracheal intubation, and anaesthesia was maintained with $2 \%$ sevoflurane in $50 \%$ air/ oxygen mixture adapted to maintain BIS values between 40 and 50. All patients had volume-controlled ventilation with a tidal volume of $6-8 \mathrm{ml} / \mathrm{kg}$ and positive end-expiratory pressure of $5 \mathrm{CmH}_{2} \mathrm{O}$.

Intraoperative data were recorded real-time in a paper case report form (CRF) and included systolic arterial pressure (SAP), diastolic arterial pressure (DAP), mean arterial pressure (MAP), heart rate, BIS, dose of ephedrine (if given), and the volume of fluids during the induction. We calculated the duration of PIH as the time span (minutes) between the defined blood pressure decrease and return to at least baseline values.

\section{Postinduction hypotension definition}

We defined $\mathrm{PIH}$ as the blood pressure decrease occurring during the first 10 minutes after induction of anaesthesia and orotracheal intubation and before surgical incision. We used several SAP and MAP thresholds for defining hypotension. Both absolute thresholds and thresholds relative to a baseline blood pressure were chosen based on the most frequently used definitions: absolute thresholds - SAP < 100, < 90, <80, $<70 \mathrm{~mm} \mathrm{Hg}$, MAP $<70,<60,<50,<40 \mathrm{~mm} \mathrm{Hg}$, and relative thresholds - SAP/MAP decrease $>10,>20,>30$, $>40 \%$ from baseline. The baseline blood pressure was defined as the SAP and MAP determined when the Cf-PWV was assessed.

\section{Statistical analysis}

All analyses were performed using Stata SE software version 13 (Stata Statistical Software: Release 12. College Station, TX: StataCorp LP). Continuous data are presented as mean \pm standard deviation (SD) and median with interquartile range (IQR), and categorical data as number and percentage frequency. The distribution of the variables was assessed using the Shapiro-Wilk test.

Logistic and linear regression analysis were used to assess the relationship between Cf-PWV and $\mathrm{PIH}$ and duration of $\mathrm{PIH}$, respectively. For the multivariable analysis we selected a priori, based on existing knowledge and literature, the following confounders: age, gender, ASA physical status, comorbidities (CAD, blood hypertension, diabetes mellitus, and malignancy), and medication ( $\beta$-blocker and ARB/ACEI use). When needed, we performed bootstrapping validation in order to avoid the problem of overfitting owing to the low number outcomes (PIH at different thresholds). For the significant associations, we used the Youden index to find the optimal cut-off for Cf-PWV for predicting PIH. A 2-tailed $p<0.05$ was considered statistically significant.

Due to the study design and to the fact that we included all the eligible patients during the study inclusion period, a power size calculation was not performed.

\section{Results}

\section{Study population characteristics}

Our study included 115 patients. Out of 121 enrolled patients, 5 were excluded due to the poor BIS signal quality and 1 due to protocol violation 
Table I. Baseline characteristics of the study population

\begin{tabular}{|c|c|}
\hline Parameter & All patients $(n=115)$ \\
\hline Age [years] & $58.1 \pm 11.4$ \\
\hline Male, $n(\%)$ & $35(30.4)$ \\
\hline BMI $\left[\mathrm{kg} / \mathrm{m}^{2}\right]$ & $27.8 \pm 5.2$ \\
\hline Baseline MAP [mm Hg] & $108.0 \pm 12.7$ \\
\hline Baseline SAP [mm Hg] & $148.5 \pm 18.9$ \\
\hline Baseline DAP [mm Hg] & $87.8 \pm 11.4$ \\
\hline \multicolumn{2}{|l|}{ ASA score, $n(\%)$} \\
\hline I & $20(17.4)$ \\
\hline$\| / I I \mid$ & $95(82.6)$ \\
\hline CAD, $n(\%)$ & $6(5.2)$ \\
\hline Blood hypertension, $n(\%)$ & $35(30.4)$ \\
\hline Diabetes mellitus, $n(\%)$ & $10(8.7)$ \\
\hline Chemotherapy, $n$ (\%) & $22(19.1)$ \\
\hline Radiotherapy, $n$ (\%) & $23(20.0)$ \\
\hline Malignancy, $n$ (\%) & $74(64.4)$ \\
\hline Haemoglobin [g/dl] & $12.6 \pm 1.7$ \\
\hline $\mathrm{EGFR}\left[\mathrm{ml} / \mathrm{min} / 1.73 \mathrm{~m}^{2}\right]$ & $71.7 \pm 13.0$ \\
\hline Sodium [mmol/l] & $143.4 \pm 2.6$ \\
\hline Potassium [mmol/l] & $4.4 \pm 0.4$ \\
\hline Serum glucose $[\mathrm{mg} / \mathrm{dl}]$ & $98.0(92.0-109.0)$ \\
\hline Cf-PWV $[\mathrm{m} / \mathrm{s}]$ & $8.0(6.7-9.1)$ \\
\hline Beta-blocker use, $n$ (\%) & $21(18.3)$ \\
\hline ARB/ACEI use, $n(\%)$ & $19(16.5)$ \\
\hline Diuretic use, $n$ (\%) & $10(8.7)$ \\
\hline $\begin{array}{l}\text { Data are expressed as mea } \\
\text { with interquartile range, or nun } \\
\text { appropriate. BMI-body mass ind } \\
\text { SAP-systolic blood pressure, DA } \\
\text { - American Society of Anesthes } \\
\text { disease, eGFR - estimated glom } \\
\text { carotid-femoral pulse wave veloc } \\
\text { blocker, ACEI-angiotensin-conve }\end{array}$ & $\begin{array}{l}\text { tandard deviation, median } \\
\text { with percent frequency, as } \\
\text { MAP-mean arterial pressure, } \\
\text { diastolic blood pressure, ASA } \\
\text { gists, CAD - coronary artery } \\
\text { lar filtration rate, Cf-PWV - } \\
\text { ARB - angiotensin II receptor } \\
\text { g-enzyme inhibitor. }\end{array}$ \\
\hline
\end{tabular}

(rapid sequence induction). The baseline characteristics of the study population are presented in Table I. The mean age was 58.1 years, $30.4 \%$ were men, and more than $80 \%$ had an ASA score of at least II (Table I). The median Cf-PWV value was $8 \mathrm{~m} / \mathrm{s}$ (IQR $6.7-9.1 \mathrm{~m} / \mathrm{s})$. The induction dose of anaesthetics is shown in Supplementary Table I.

\section{Carotid-to-femoral pulse wave velocity and postinduction hypotension}

In the univariable analysis, Cf-PWV was associated with the development of PIH when defined as a relative SAP decrease of $>30 \%$ and $40 \%$ from baseline, absolute MAP $<50 \mathrm{~mm} \mathrm{Hg}$, and as relative MAP decrease of $>40 \%$ from baseline (see Table II). In the multivariable analysis, with the exception of absolute MAP, all these associations remained statistically significant $(\mathrm{OR}=1.77$, 95\% Cl: 1.09-2.86, OR $=1.31$, 95\% Cl: 1.01-1.69, and $\mathrm{OR}=1.52,95 \% \mathrm{Cl}: 1.15-1.99$ for SAP decrease of $>30 \%$ and of $>40 \%$ from baseline and for the relative MAP decrease of $>40 \%$ from baseline, respectively - see Table II).

The following Cf-PWV thresholds with their respective sensitivities and specificities were identified for each of the 3 significant associations: $7.25 \mathrm{~m} / \mathrm{s}(95 \% \mathrm{Cl}: 5.79-8.71 \mathrm{~m} / \mathrm{s})$, sensitivity $66 \%$, specificity $71 \% ; 8.05 \mathrm{~m} / \mathrm{s}(95 \% \mathrm{Cl}: 6.36-9.74 \mathrm{~m} / \mathrm{s})$, sensitivity $56 \%$, specificity $67 \%$; and $8.05 \mathrm{~m} / \mathrm{s}$ (95\% Cl: 6.33-9.77), sensibility $58 \%$, specificity $68 \%$ for predicting PIH defined as relative SAP decrease of $>30 \%$ and $40 \%$ from baseline and as relative MAP decrease of $>40 \%$ from baseline, respectively.

\section{Carotid-to-femoral pulse wave velocity and duration of postinduction hypotension}

As shown in Table III, Cf-PWV was positively associated with duration of $\mathrm{PIH}$ when defined as SAP decrease of $>20 \%,>30 \%$, and $>40 \%$ from baseline or as MAP decrease of $>30 \%$ and $>40 \%$ from baseline. However, when adjusting for age, gender, ASA score, comorbidities, and chronic medication, Cf-PWV remained significantly associated only with duration of PIH if defined as an SAP or MAP decrease of $>40 \%$ from baseline ( $\beta$ coefficient $0.45,95 \% \mathrm{Cl}: 0.01-0.88$ and $0.49,95 \% \mathrm{Cl}$ : 0.08-0.89, respectively - see Table III).

\section{Discussion}

In this study, we investigated the association between arterial stiffness and severity and duration of PIH. We found that arterial stiffness evaluated by Cf-PWV identifies patients at risk of severe $\mathrm{PIH}$, independently of age, gender, ASA score, comorbidities, and chronic medication and higher the Cf-PWV value, more pronounced decrease of SAP or MAP.

Maintaining haemodynamic stability during anaesthesia is essential for reducing the rate of postoperative complications. Prevention of hypotensive events plays a key role in providing patient safety [17].

Hypotension can occur at various times during anaesthesia, with different causes and with varying consequences. $\mathrm{PIH}$, which occurs between anaesthesia induction and surgical incision, is a largely preventable consequence of anaesthesia [4], being a potentially modifiable risk factor. Induction to general anaesthesia causes decreased 
Table II. The relationship between pulse wave velocity $(\mathrm{m} / \mathrm{s})$ and postinduction hypotension $(\mathrm{PIH})$ using different thresholds

\begin{tabular}{|c|c|c|c|c|}
\hline PIH thresholds & $\mathrm{OR}^{*}(95 \% \mathrm{Cl})$ & $P$-value & Adjusted $^{* *} \mathrm{OR}^{*}(95 \% \mathrm{Cl})$ & $P$-value \\
\hline \multicolumn{5}{|c|}{ Absolute SAP [mm Hg] } \\
\hline$<100$ & $0.906(0.669-1.228)$ & 0.53 & $0.663(0.390-1.128)$ & 0.13 \\
\hline$<90$ & $0.974(0.787-1.207)$ & 0.81 & $0.866(0.622-1.207)$ & 0.39 \\
\hline$<80$ & $1.101(0.881-1.375)$ & 0.39 & $1.039(0.738-1.462)$ & 0.83 \\
\hline$<70$ & $1.231(0.927-1.635)$ & 0.15 & $1.109(0.701-1.754)$ & 0.44 \\
\hline \multicolumn{5}{|c|}{ Relative SAP thresholds, decrease from baseline (\%) } \\
\hline$>10$ & NA & NA & NA & NA \\
\hline$>20$ & $1.890(0.350-10.199)$ & 0.46 & $2.368(0.269-20.843)$ & 0.44 \\
\hline$>30$ & $1.738(1.104-2.734)$ & 0.02 & $1.767(1.093-2.856)$ & 0.02 \\
\hline$>40$ & $1.271(1.025-1.578)$ & 0.04 & $1.309(1.010-1.698)$ & 0.04 \\
\hline \multicolumn{5}{|c|}{ Absolute MAP [mm Hg] } \\
\hline$<70$ & $0.939(0.739-1.195)$ & 0.61 & $0.946(0.658-1.361)$ & 0.77 \\
\hline$<60$ & $1.059(0.849-1.321)$ & 0.61 & $1.056(0.734-1.519)$ & 0.77 \\
\hline$<50$ & $1.445(1.033-2.021)$ & 0.03 & $1.050(0.619-1.783)$ & 0.86 \\
\hline$<40$ & NA & NA & NA & NA \\
\hline \multicolumn{5}{|c|}{ Relative MAP thresholds, decrease from baseline (\%) } \\
\hline$>10$ & NA & NA & NA & NA \\
\hline$>20$ & $2.355(0.606-9.145)$ & 0.22 & $3.196(0.569-17.964)$ & 0.19 \\
\hline$>30$ & $1.121(0.823-1.526)$ & 0.47 & $1.274(0.903-1.796)$ & 0.17 \\
\hline$>40$ & $1.377(1.086-1.746)$ & 0.008 & $1.516(1.154-1.991)$ & 0.003 \\
\hline
\end{tabular}

${ }^{\star} O R$ are per $1 \mathrm{~m} / \mathrm{s}$ increase in the pulse wave velocity. ${ }^{* *}$ Adjusted for age, gender, ASA score, comorbidities, and chronic medication. $P I H$ - postinduction hypotension, $\mathrm{Cl}$ - confidence interval, OR - odds ratio, SAP - systolic arterial pressure, MAP - mean arterial pressure, NA - not applicable.

blood pressure via various mechanisms, such as anaesthetic effects on blood vessel walls leading to vasodilatation and a drop in systemic vascular resistance [18]. Propofol has well-known potential to induce hypotension by several mechanisms, the most important one being vasodilatation [19]. Thus, propofol is recognized as an independent risk factor for hypotension [5]. Propofol is the most commonly used anaesthesia induction agent in the world [20]. We used sevoflurane as the inhalational anaesthetic because it has been demonstrated to have the lowest morbidity and mortality of currently used inhaled anaesthetics and has the least pronounced cardiovascular effect [21]. Also, we protocolized induction of anaesthesia and included BIS monitoring to provide study group homogeneity in terms of depth of anaesthesia. Reich et al. described the occurrence of severe hypotension, defined as a MAP decrease of more than $40 \%$ from baseline, in $9 \%$ of subjects during the first 10 minutes after induction of general anaesthesia during non-cardiac surgery.
Risk factors for hypotension were identified as ASA III-IV, age over 50 years, baseline MAP under $70 \mathrm{~mm} \mathrm{Hg}$, use of propofol at induction, and high doses of fentanyl (>5 $\mu \mathrm{g} / \mathrm{kg}$ ) [5].

Angiotensin-converting enzyme inhibitors/ARBs were discontinued on the day before the surgery because perioperative use of ACEIs or ARBs carries a risk of severe hypotension under anaesthesia, in particular following induction of general anaesthesia $[22,23]$. It is recommended that ACEIs/ARBs are withheld $24 \mathrm{~h}$ before surgery with the attention to restart the medication within $48 \mathrm{~h}$ after surgery [23]. The continuation or cessation of diuretics should be considered on a patient-by-patient basis $[22,23]$. We stopped diuretics to all patients before surgery because there is a risk of hypovolaemia and subsequent increased risk of $\mathrm{PIH}$ in patients treated with diuretics.

Prediction of hypotension at induction of general anaesthesia based on the variables available to the clinician before induction of anaesthesia remains a difficult task. Studies that evaluated risk 
Table III. The relationship between pulse wave velocity $(\mathrm{m} / \mathrm{s})$ and duration of postinduction hypotension $(\mathrm{PIH})$ (minutes) using different thresholds

\begin{tabular}{|c|c|c|c|c|}
\hline PIH thresholds & $\beta$ Coefficient* $(95 \% \mathrm{Cl})$ & $P$-value & Adjusted $^{* *} \beta$ coefficient $^{*}(95 \% \mathrm{Cl})$ & $P$-value \\
\hline \multicolumn{5}{|c|}{ Absolute SAP [mm Hg] } \\
\hline$<100$ & $-0.098(-0.499$ to 0.304$)$ & 0.63 & $-0.076(-0.676$ to 0.524$)$ & 0.80 \\
\hline$<90$ & $0.066(-0.245$ to 0.376$)$ & 0.68 & $-0.046(-0.512$ to 0.419$)$ & 0.85 \\
\hline$<80$ & 0.089 (-0.064 to 0.243$)$ & 0.25 & $0.099(-0.135$ to 0.333$)$ & 0.40 \\
\hline$<70$ & $0.042(-0.028$ to 0.112$)$ & 0.24 & $0.023(-0.083$ to 0.129$)$ & 0.67 \\
\hline \multicolumn{5}{|c|}{ Relative SAP, decrease from baseline (\%) } \\
\hline$>10$ & $0.063(-0.165$ to 0.291$)$ & 0.59 & $0.212(-0.130$ to 0.555$)$ & 0.22 \\
\hline$>20$ & 0.313 (0.004 to 0.622$)$ & 0.04 & $0.356(-0.095$ to 0.807$)$ & 0.12 \\
\hline$>30$ & 0.622 (0.229 to 1.015$)$ & 0.002 & $0.491(-0.084$ to 1.065$)$ & 0.09 \\
\hline$>40$ & 0.579 (0.276 to 0.882$)$ & $<0.001$ & 0.445 (0.007 to 0.884$)$ & 0.04 \\
\hline \multicolumn{5}{|c|}{ Absolute MAP [mm Hg] } \\
\hline$<70$ & $-0.057(-0.399$ to 0.285$)$ & 0.74 & $-0.020(-0.500$ to 0.459$)$ & 0.93 \\
\hline$<60$ & $0.053(-0.112$ to 0.218$)$ & 0.52 & $0.032(-0.201$ to 0.264$)$ & 0.79 \\
\hline$<50$ & $0.041(-0.009$ to 0.091$)$ & 0.11 & $0.008(-0.066$ to 0.082$)$ & 0.83 \\
\hline$<40$ & NA & NA & NA & NA \\
\hline \multicolumn{5}{|c|}{ Relative MAP, decrease from baseline (\%) } \\
\hline$>10$ & NA & NA & NA & NA \\
\hline$>20$ & $0.181(-0.132$ to 0.493$)$ & 0.25 & $0.427(-0.027$ to 0.881$)$ & 0.07 \\
\hline$>30$ & 0.448 (0.049 to 0.848$)$ & 0.03 & $0.551(-0.028$ to 1.130$)$ & 0.06 \\
\hline$>40$ & $0.489(0.211$ to 0.767$)$ & 0.001 & 0.486 (0.083 to 0.889$)$ & 0.02 \\
\hline
\end{tabular}

${ }^{\star} \beta$ coefficient are per $1 \mathrm{~m} / \mathrm{s}$ increase in the pulse wave velocity. ${ }^{* *}$ Adjusted for age, gender, ASA score, comorbidities, and chronic medication. $P I H$ - postinduction hypotension, $C I$ - confidence interval, SAP-systolic arterial pressure, MAP-mean arterial pressure, NA - not applicable.

factors for $\mathrm{PIH}$ provided few risk factors that are potentially modifiable [24]. Increased arterial stiffness is associated with endothelial dysfunction and high levels of free fatty acids, g-glutamyl transferase, leptin, and endothelin-1 in the serum [25, 26]. Carotid-to-femoral pulse wave velocity was found to have a strong positive association with carotid intima-media thickness, carotid and aortic plaque presence [27], aortic and coronary calcifications [28], and coronary atherosclerosis [29]. Carotid-to-femoral pulse wave velocity predicts cardiovascular outcomes above and beyond traditional cardiovascular risk factors (blood hypertension, chronic kidney disease, diabetes mellitus) [14]. Nakano et al. showed that patients with a Cf-PWV higher than $10 \mathrm{~m} / \mathrm{s}$ have a $30 \%$ higher risk of cerebrovascular disease [30]. Also, after evaluating 710 patients with essential hypertension, Blacher et al. found that a PWV higher than $13 \mathrm{~m} / \mathrm{s}$ is a strong predictor of cardiovascular mortality [31]. None of the above cardiovascular risk factors associated with increased arterial stiff- ness are evaluated routinely preoperatively, which means that determining preoperative Cf-PWV could improve cardiovascular risk stratification. In 2007 ESH/ESC hypertension guidelines proposed a fixed threshold value for Cf-PWV (12 m/s) based on published epidemiological studies [32]. Currently, the reference value for normal Cf-PWV is highly variable, being dependent on the studied population and in the absence of Cf-PWV measurement standardization [33, 34].

In our study, preoperative assessment of Cf-PWV was able to identify patients at risk of developing severe hypotension defined as SAP or MAP decrease of more than $40 \%$ from baseline. Also, Cf-PWV was significantly associated with the duration of PIH as defined as above. Alecu et al. investigated the relationship between CfPWV and variations in arterial pressure during induction of anaesthesia [15]. Compared to our study, their population was older (mean age 71.1 vs. 58.1 years) and had higher Cf-PWV (12.1 vs. $8 \mathrm{~m} / \mathrm{s})$, which means that preoperative Cf-PWV 
assessment can identify a subpopulation of patients (younger and apparently healthier) that have an increased risk of $\mathrm{PIH}$. Also, their results found that increased Cf-PWV $>12.9 \mathrm{~m} / \mathrm{s}$ is associated with a decrease in SAP of more than $25 \%$ of baseline. Our study investigated the decrease in arterial pressure using multiple definitions of $\mathrm{PIH}$ known to result in increased postoperative morbidity and mortality [3, 29, 30].

Our study did not intend to quantify PIH effects on clinical outcome variables, because it was designed to evaluate whether preoperative arterial stiffness assessment could identify patients at increased risk of $\mathrm{PIH}$. In the literature, there is strong evidence to support the relationship between $\mathrm{IOH}$ and increased postoperative morbidity and mortality after non-cardiac surgery $[3,6]$. Also, how to characterize $\mathrm{IOH}$ remains unclear, and there is no universal definition for hypotension. In order to assess $\mathrm{IOH}$ secondary organ injury, researchers use most frequently absolute or relative MAP or SAP thresholds. The Perioperative Quality Initiative consensus statement on intraoperative blood pressure, risk, and outcomes for elective surgery stated that intraoperative mean arterial blood pressure below $60-70 \mathrm{~mm} \mathrm{Hg}$ or a SAP below $100 \mathrm{~mm} \mathrm{Hg}$ is associated with myocardial injury, acute kidney injury, and death, injury being a function of hypotension severity and duration [23]. Bijker et al. defined $\mathrm{IOH}$ as a decrease in MAP of more than $30 \%$ from the preoperative blood pressure. Their results showed that $\mathrm{IOH}$ accounts for an increase in stroke risk of approximately $1.3 \%$ per minute of hypotension [3]. Salmasi et al. determined that a MAP of $50 \mathrm{~mm} \mathrm{Hg}$ for just 1 minute significantly increases the risk for myocardial and kidney injury. Also, when MAP was more than $50 \%$ below the preoperative values for just 5 minutes, the risk for both myocardial and kidney injury increased significantly [35]. Acute kidney injury occurs in approximately 7.5\% of patients who undergo noncardiac surgery [36]. Small changes in serum creatinine are increasingly recognized as strong independent risk factors for short- and long-term mortality [37] and more costly health care after surgery [38]. Also, myocardial injury occurs in $11.6 \%$ of noncardiac surgery and is also associated with a strong, independent risk of death after surgery, even with only small biomarker increases [39].

Even though arterial blood pressure monitoring at least every 5 minutes is a standard monitoring practice [40], there are no guidelines on blood pressure values to be maintained during surgery [6]. Futier et al. demonstrated that patients undergoing general anaesthesia, who had an individualized strategy aimed at achieving an intraoperative SAP within $10 \%$ of the ref- erence value (resting blood pressure assessed during the preoperative anaesthesiology consultation), had significantly lower rates of organ disfunction after surgery [41].

Although the identified sensibilities and specificities for Cf-PWV cut-offs are relatively low, it should be noted that their value is derived from a statistical technique that defines the maximum potential effectiveness of a parameter, giving equal weight to sensitivity and specificity $[42,43]$. With these limitations in mind, we believe that it is of great importance that the Cf-PWV levels are independently associated with $\mathrm{PIH}$, and also that these cut-offs should be tested for relevance in future prospective studies.

Strengths and limitations. Aiming to describe the correlation between arterial stiffness and $\mathrm{PIH}$, our study design excluded patients having known risk factors for $\mathrm{PIH}$. Thus, our study design strongly supports the idea that preoperative Cf-PWV assessment can identify a subpopulation of patients without known risk factors (younger and apparently healthier), who have an increased risk of $\mathrm{PIH}$. Also, it protocolized patient preanesthetic preparation and technique and monitoring of anaesthesia induction in order to exclude confounders. Moreover, we explored blood pressure decline from baseline, using several blood pressure thresholds, in absolute and relative values, for both SAP and MAP because there is no universal definition for $\mathrm{IOH}$. There are inherent limitations in the ability to generalize our findings, being a single-centre study, including a small number of patients with a high prevalence of cancer patients, and assessing hypotension during a limited time span (10 minutes between induction and surgical incision). Furthermore, even though the population of hypertensive patients was small (30.4\%), the increased baseline MAP, SAP, and DAP may signify that there were patients undertreated or with no treatment for hypertension. Thus, our results need to be validated by larger multi-centric studies.

In conclusion, preoperative assessment of arterial stiffness identifies patients at risk of pronounced decrease in blood pressure during induction of general anaesthesia, even in patients without known risk factors. Carotid-to-femoral pulse wave velocity is a simple, non-invasive, lowcost bedside technique, which may prove to be a useful tool for perioperative risk assessment. Further prospective research is required to clarify the prognostic significance of vascular stiffness in patients undergoing general anaesthesia.

\section{Acknowledgments}

This work was supported by a grant from the Ministry of Research and Innovation, CNCS-UEFISCDI, project number PN-III-P1-1.1-PD-2016-0287. 


\section{Conflict of interest}

The authors declare no conflict of interest.

\section{References}

1. Bijker JB, Van Klei WA, Kappen TH, Van Wolfswinkel L, Moons KGM, Kalkman CJ. Incidence of intraoperative hypotension as a function of the chosen definition: Literature definitions applied to a retrospective cohort using automated data collection. Anesthesiology 2007; 107: 213-20.

2. Walsh M, Devereaux PJ, Garg AX, et al. Relationship between intraoperative mean arterial pressure and clinical outcomes after noncardiac surgery: toward an empirical definition of hypotension. Anesthesiology 2013; 119: 507-15.

3. Bijker JB, Persoon S, Peelen LM, et al. Intraoperative hypotension and perioperative ischemic stroke after general surgery, a nested case-control study. Anesthesiology 2012; 116: 658-64.

4. Maheshwari K, Turan A, Mao G, et al. The association of hypotension during non-cardiac surgery, before and after skin incision, with postoperative acute kidney injury: a retrospective cohort analysis. Anaesthesia 2018; 73: $1223-8$.

5. Reich DL, Hossain S, Krol M, et al. Predictors of hypotension after induction of general anesthesia. Anesth Analg 2005; 101: 622-8.

6. Monk TG, Bronsert MR, Henderson WG, et al. Association between intraoperative hypotension and hypertension and 30-day postoperative mortality in noncardiac surgery. Anesthesiology 2015; 123: 307-19.

7. Haynes AB, Regenbogen SE, Weiser TG, et al. Surgical outcome measurement for a global patient population: validation of the surgical Apgar score in 8 countries. Surgery 2011; 149: 519-24.

8. Vlachopoulos C, Aznaouridis K, Stefanadis C. Prediction of cardiovascular events and all-cause mortality with arterial stiffness. A systematic review and meta-analysis. J Am Coll Cardiol 2010; 55: 1318-27.

9. Gavish B, Izzo JL. Arterial stiffness: going a step beyond. Am J Hypertens 2016; 29: 1223-33.

10. Anderson TJ. Arterial stiffness or endothelial dysfunctin as a surrogate marker of vascular risk. Can J Cardiol 2006; 22 Suppl B: 72B-80B.

11. Higashi Y, Noma K, Yoshizumi M, Kihara Y. Endothelial function and oxidative stress in cardiovascular diseases. Circ J 2009; 73: 411-8.

12. de Rezende Mikael L, de Paiva AMG, Gomes MM, et al. Vascular aging and arterial stiffness. Arq Bras Cardiol 2017; 109: 253-8.

13. Laurent S, Cockcroft J, Van Bortel L, et al. Expert consensus document on arterial stiffness: methodological issues and clinical applications. Eur Heart J 2006; 27: 2588-605.

14. Laurent $S$, Boutouyrie P, Asmar R, et al. Aortic stiffness is an independent predictor of all-cause and cardiovascular mortality in hypertensive patients. Hypertension 2001; 37: 1236-41.

15. Alecu C, Cuignet-Royer E, Mertes PM, et al. Pre-existing arterial stiffness can predict hypotension during induction of anaesthesia in the elderly. Br J Anaesth 2010; 105: 583-8.

16. Nakata Y, Goto T, Ishiguro Y, Terui K, Niimi Y, Morita S. Preoperative pulse wave velocity fails to predict hemodynamic responses to anesthesia and to surgical stimulation. J Clin Anesth 1999; 11: 285-9.
17. Cheung CC, Martyn A, Campbell N, et al. Predictors of intraoperative hypotension and bradycardia. Am J Med 2015; 128: 532-8.

18. Robinson BJ, Ebert TJ, O'Brien TJ, Colinco MD, Muzi M. Mechanism whereby propofol mediates peripheral vasodilation in humans. Sympathoinhibition or direct vascular relaxation? Anesthesiology 1997; 86: 64-72.

19. Billard V, Moulla F, Bourgain JL, Megnigbeto A, Stanski DR. Hemodynamic response to induction and intubation: propofol/fentanyl interaction. Anesthesiology 1994; 81: 1384-93.

20. Eckenhoff $R$, Tang P. Recent progress on the molecular pharmacology of propofol. F1000Res 2018; 7: 123.

21. Eis $S$, Kramer J. Anesthesia inhalation agents cardiovascular effects. StatPearls [Internet] 2019.

22. Fleisher LA, Fleischmann KE, Auerbach AD, et al. 2014 ACC/AHA guideline on perioperative cardiovascular evaluation and management of patients undergoing noncardiac surgery: a report of the American College of Cardiology/American Heart Association Task Force on practice guidelines. J Am Coll Cardiol 2014; 64: e77-137.

23. Sessler DI, Bloomstone JA, Aronson S, et al. Perioperative quality initiative consensus statement on intraoperative blood pressure, risk and outcomes for elective surgery. Br J Anaesth 2019; 122: 563-74.

24. Südfeld S, Brechnitz S, Wagner JY, et al. Post-induction hypotension and early intraoperative hypotension associated with general anaesthesia. Br J Anaesth 2017; 119: 57-64.

25. Kozakova M, Natali A, Dekker J, et al. Insulin sensitivity and carotid intima-media thickness: relationship between insulin sensitivity and cardiovascular risk study. Arterioscler Thromb Vasc Biol 2013; 33: 1409-17.

26. Kozakova M, Palombo C, Paterni Eng M, et al. Fatty liver index, gamma-glutamyltransferase, and early carotid plaques. Hepatology 2012; 55: 1406-15.

27. Van Popele NM, Grobbee DE, Bots ML, et al. Association between arterial stiffness and atherosclerosis: the Rotterdam Study. Stroke 2001; 32: 454-60.

28. McEniery CM, McDonnell BJ, So A, et al. Aortic calcification is associated with aortic stiffness and isolated systolic hypertension in healthy individuals. Hypertension 2009; 53: 524-31.

29. Oberoi S, Schoepf UJ, Meyer M, et al. Progression of arterial stiffness and coronary atherosclerosis: longitudinal evaluation by cardiac CT. Am J Roentgenol 2013; 200: 798-804.

30. Nakano H, Okazaki K, Ajiro Y, Suzuki T, Oba K. Clinical usefulness of measuring pulse wave velocity in predicting cerebrovascular disease: evaluation from a cross-sectional and longitudinal follow-up study. J Nippon Med Sch 2001; 68: 490-7.

31. Blacher J, Asmar R, Djane S, London GM, Safar ME. Aortic pulse wave velocity as a marker of cardiovascular risk in hypertensive patients. Hypertension 1999; 33: 1111-7.

32. Mancia G, De Backer G, Dominiczak A, et al. 2007 guidelines for the management of arterial hypertension: the task force for the management of arterial hypertension of the European Society of Hypertension (ESH) and of the European Society of Cardiology (ESC). J Hypertens 2007; 25: 1105-87.

33. Mattace-Raso FUS, Hofman A, Verwoert GC, et al. Determinants of pulse wave velocity in healthy people and in the presence of cardiovascular risk factors: 'Establishing normal and reference values'. Eur Heart J 2010; 31: 2338-50.

34. Brunoni AR, Szlejf C, Suemoto CK, et al. Association between ideal cardiovascular health and depression 
incidence: a longitudinal analysis of ELSA-Brasil. Acta Psychiatr Scand 2019; 140: 552-62.

35. Salmasi V, Maheshwari K, Yang D, et al. Relationship between intraoperative hypotension, defined by either reduction from baseline or absolute thresholds, and acute kidney and myocardial injury after noncardiac surgery. Anesthesiology 2017; 126: 47-65.

36. Abelha FJ, Botelho M, Fernandes V, Barros H. Determinants of postoperative acute kidney injury. Crit Care 2009; 13: 79.

37. Bellomo R, Kellum JA, Ronco C. Defining and classifying acute renal failure: From advocacy to consensus and validation of the RIFLE criteria. Intensive Care Med 2007; 33: 409-13.

38. Chertow GM, Burdick E, Honour M, Bonventre JV, Bates DW. Acute kidney injury, mortality, length of stay, and costs in hospitalized patients. J Am Soc Nephrol 2005; 16: 3365-70.

39. Devereaux PJ, Biccard BM, Sigamani A, et al. Association of postoperative high-sensitivity troponin levels with myocardial injury and 30-day mortality among patients undergoing noncardiac surgery. J Am Med Assoc 2017; 317: 1642-51.

40. Standards for Basic Anesthetic Monitoring | American Society of Anesthesiologists (ASA) [Internet]. Available from: https://www.asahq.org/standards-and-guidelines/ standards-for-basic-anesthetic-monitoring.

41. Futier E, Lefrant JY, Guinot PG, et al. Effect of individualized vs standard blood pressure management strategies on postoperative organ dysfunction among high-risk patients undergoing major surgery: a randomized clinical trial. J Am Med Assoc 2017; 318: 1346-57.

42. Reiser B. Measuring the effectiveness of diagnostic markers in the presence of measurement error through the use of ROC curves. Stat Med 2000; 19: 2115-29.

43. Faraggi $D$. The effect of random measurement error on receiver operating characteristic (ROC) curves. Stat Med 2000; 19: 61-70. 